\title{
Modeling spatial patterns in the visual cortex
}

\author{
Yudy Carolina Daza C.," Carolina B. Tauro, and Francisco A. Tamarit \\ FAMAF, Instituto de Física Enrique Gaviola, Universidad Nacional de Córdoba, Argentina \\ Pablo M. Gleiser \\ Statistical and Interdisciplinary Physics Group, Centro Atómico Bariloche, Argentina
}

(Received 11 June 2014; published 29 October 2014)

\begin{abstract}
We propose a model for the formation of patterns in the visual cortex. The dynamical units of the model are Kuramoto phase oscillators that interact through a complex network structure embedded in two dimensions. In this way the strength of the interactions takes into account the geographical distance between units. We show that for different parameters, clustered or striped patterns emerge. Using the structure factor as an order parameter we are able to quantitatively characterize these patterns and present a phase diagram. Finally, we show that the model is able to reproduce patterns with cardinal preference, as observed in ferrets.
\end{abstract}

DOI: 10.1103/PhysRevE.90.042818

PACS number(s): $89.75 . \mathrm{Fb}, 87.18 . \mathrm{Hf}, 89.75 . \mathrm{Hc}$

\section{INTRODUCTION}

There are many examples of systems, both natural and artificial, which present complex global behaviors even though they are composed by relatively simple units [1-4]. In many cases the origin of such a global complexity resides on the intricate interaction network between the units [5]. Nowadays we know that many of these networks have particular, but at the same time universal, topological properties: on the one hand the connectivity per unit histogram is very broad, and can be approximated by a power-law (scale free networks) [6]; on the other hand, although the mean-free path between units is relatively short, the clustering is unusually large (small world networks) [7]. That is why during the last decade the statistical mechanics community has put particular attention on describing the topological properties of these networks in order to find out universal qualities, generating more realistic models that take into account their development and evolution, and even studying the behavior of a variety of models whose interactions are ruled by these kind of networks [5,8-11].

One distinctive feature of most real systems is that they are embedded in a Euclidean space, where it is possible to spatially localize their constituents, and where the usual notion of distance between units is valid. In topographic terms, these systems are embedded in a low-dimensional Euclidean space. However, most of the scale free and small world models do not take into account the physical distance between units. In many cases this simplification is not realistic, and there are a lot of examples of natural and artificial systems in which the Euclidean distance play a fundamental role in both the development and the performance of the network.

In this work, we will focus in the neocortex of mammalian brains, that is a complex system and also is embedded in a low dimensional Euclidean space [12-15]. Furthermore, it is a thin layer of cells covering most of the brain surface. The visual cortex or primary visual cortex (or area 17, area V1

\footnotetext{
*ycdazac@famaf.unc.edu.ar; Also at Statistical and Interdisciplinary Physics Group, Centro Atómico Bariloche, Argentina.
}

or striate cortex [16]) is the region that captures elements of the natural scene, such as contours, textures, colors, shadows, movements, and disparities. Also, it is organized in functional modules, in such a way that neurons with similar receptive fields form vertically oriented structures called columns. At the same time, different columns are connected horizontally between them. One of these are the so-called orientation preference columns, composed by neurons which are activated by stimulus of the same orientation. When certain stimulus is presented, such as a bar with a specific orientation, it is possible to observe in vivo the orientational pattern formed by active and non-active neurons [17]. Even though the cortex can be divided histologically into six cell layers, we will consider only a single layer, and consider only inter-layer interactions in a bi-dimensional structure. As we will show, even with this simplification we obtain patterns as observed in experiments in ferrets [18], macaques [19], and monkeys [20]. In this article we present a model inspired by the mammalian visual cortex, that is formed by a set of Kuramoto phase oscillators $[3,21,22]$ which interact in a complex network. By embedding this network in two-dimensional Euclidean space we are able to define distance dependent interactions. Using numerical simulations we study the different synchronization patterns that emerge as a function of the parameters of the model. We find that the model allows for the emergence of clustered and striped phases. We show that for certain parameters, these patterns resemble the orientational preference columns patterns observed experimentally in the striate cortex [18-20].

\section{THE MODEL}

The patterns observed in visual cortex maps clearly emerge from the dynamics of a great number of elements that have non-linear interactions. These elements are a heterogeneous neuronal population whose interactions consists generally in the excitation of others neurons or the inhibition of their activity. Also, through their interactions the neurons are able to synchronize their activity. The interactions between the neurons takes place in a complex network, that is embedded in a low-dimensional Euclidean space. In this section we present a model in order to understand which are the main 
mechanisms and properties of the cortex that allow for the emergence of self-organized maps. The model accounts for the following elements: a specific dynamic for the neural units, linked by a complex network of connections, interacting in a non-linearly way and able to synchronize their activity. We follow with a brief description of each ingredient of the model.

First, we consider the dynamics of the neural units. It is known that several activities of the neuron can be modeled by periodical processes and that the neurons are capable to influence one another by electrical or chemical signals. Through this mechanisms the neurons adjust their activity to the activity of others neurons in the medium. This mutual activity adjustment is the cause of the synchronization that emerges when a population of neurons, for example, are exposed to some stimulus, like oriented bars in their receptive field. The neurons increase their activity for a specific stimuli such as line or an edge with a given orientation and then, depending on their interactions, can reach some synchronized states with the other neurons in which neurons in the same cluster respond to the orientation of the line. This orientation can be represented by a phase angle. This periodical variable together with the coupling between elements leads us to consider the formalism of coupled phase oscillators [3,9,21,22]. In this way the units are represented by a phase value in the interval $[0, \pi)$.

After choosing the units we focus on the network of interactions. The neurons in the fourth layer of the cortex, that are responsible for the orientation processing [23], send mainly horizontal afferents to other neurons in a way that depends of the neuron (or its localization) and its neighbors. There is growing evidence that the structure of connections in the cortex follows a broad distribution where most units have few connections while a small number have many. We approximate this by using a power-law degree distribution (or scale-free network). An additional fact is that the neuron real afferents have a length and also a limited number of connections that depends on physical constraints of the neuron tissue in which it is located. These assumptions allows us to establish a realistic lattice of connections in which the neurons have connections with a given length, a broad degree distribution and the possibility to reach a saturation value [12]. A method of network construction that best suit these characteristics was presented by Rozenfeld et al. [24,25], in which a network with a power-law degree distribution can be embedded in an Euclidean space and the maximum distance of connections can be minimized by the variation of a parameter. In Fig. 1 we show a lattice with $N=60 \times 60$ units. We show explicitly the connections of nine units, and also in the background present a typical pattern representing the phase value of the units. In this way we can compare the scale of the connections with the emerging patterns as in the work of Stettler et al. [26]. Also, by incorporating both a scale-free network and an embedding in the complex network structure the neurons which are close have many connections, and the connections become sparse with distance [12]. This effect can be tuned with the parameters of the model to obtain extreme cases, such as a regular lattice, or a mean field interaction [27]. In this way we can compare the results of the model with known systems.

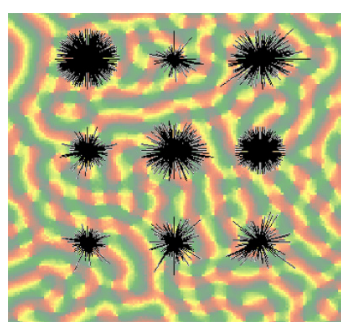

FIG. 1. (Color online) A lattice with $N=60 \times 60$ sites, showing explicitly the connections of nine units. The background presents a typical pattern representing the phase value of the units.

Now, we have the appropriate units and the lattice in which they are located, the missing ingredient is the form of the interaction function. It is expected that this will play a fundamental role in the configuration of the order-disorder states. In the visual cortex each neuron receives excitatory synaptic potentials from many afferent fibers and integrate in order to produce an action potential. Each neuron also receives inhibitory synaptic potentials that prevents the membrane potential to reaching a threshold and spike [16]. Additionally, the distribution of this synaptic potentials exhibits a particular structure: to short range $(50-100 \mu)$ are excitatory and outside of this area (until 200-500 $\mu$ ) are inhibitory [28]. This particular field of interaction resumes in the typical form of a Mexican hat, widely used to represent the synaptic interaction strength of a single neuron [29]. The Mexican hat function has two fundamental parameters: one allows for the tuning of the balance between excitatory and inhibitory interactions, while the other modulates the distance at which there is an effective interaction (Fig. 2). As we will show, the modulation of these parameters leads to different patterns of synchronization.

The dynamics of the model is summarized in the following equations [30]. The phases $\phi_{i}$ evolve according to

$$
\frac{\partial \phi_{i}}{\partial t}=-2 \epsilon \sum_{j} I\left(\vec{r}_{i}, \vec{r}_{j}\right) \sin \left(2 \phi_{j}-2 \phi_{i}\right),
$$

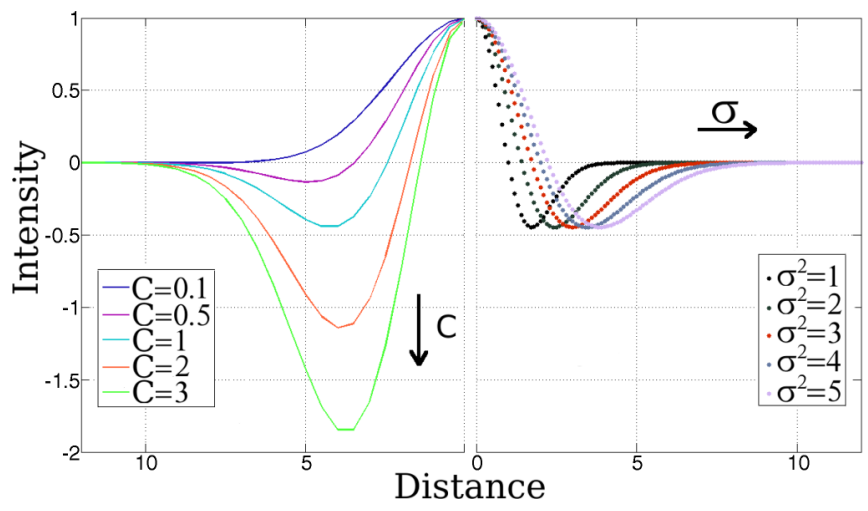

FIG. 2. (Color online) Mexican hat interaction function. Parameter $C$ modulates the balance between excitatory and inhibitory interactions, while $\sigma$ regulates the distance at which there is an effective interaction. 
where $i, j=1 \ldots N$ and $\epsilon$ is the change rate of the stimuli coming from lateral cells. The interactions are given by the Mexican hat function $I$ :

$$
I\left(\vec{r}_{i}, \vec{r}_{j}\right)=\left(1-C \frac{\left(\vec{r}_{i}-\vec{r}_{j}\right)^{2}}{\sigma^{2}}\right) \exp \left(\frac{\left|\vec{r}_{i}-\vec{r}_{j}\right|^{2}}{2 \sigma^{2}}\right) .
$$

We use the method proposed by Rozenfeld et al. [24,25] to embed a power-law degree distribution of connections in a Euclidean lattice.

\section{RESULTS}

The numerical simulations of the time evolution of the phases are made by means of the Euler numerical integration method. The initial states are phases uniformly spread in the $[0, \pi)$ interval. The network parameters are: the exponent $\alpha$ of the power-law degree distribution, and the minimum $(m)$ and maximum $(M)$ connectivities. Parameter $A$ regulates the maximum geographical distance at which a site can connect, thus acting as a saturation control parameter. We use periodic boundary conditions and present a graphical representation of the phases consisting in colors which encode their preferred stimulus orientation.

Tauro et al. [27] recently analyzed the effects of embedding a scale-free network in the synchronization of phase oscillators. They observed that this network structure allows for the emergence of multiple clusters synchronized at different frequencies. In our model the presence of competing interactions through the Mexican hat function allows for the emergence of new patterns with a striped structure. Note that now qualitative changes in the different patterns can be tuned both through the network structure or the interaction function. For example increasing parameter $\sigma$, that regulates the distance at which there is an effective interactions, leads to qualitative changes in the striped structure, as can be seen by comparing Figs. 3(a) and 3(b). On the other hand, shifting the embedding parameters towards smaller values or increasing the scale-free exponent $\alpha$ limits the number of connections available to hubs, and clustered structures emerge [27].

In Fig. 3 we show the time evolution of a system with $N=$ $60 \times 60$ oscillators, for four different sets of parameters. In all cases the system evolves from initial aleatory conditions until it reaches a stationary state. A number of different emerging patterns can be observed, such as striped phases as in Fig. 3(a) and (b), or clustered phases as in Fig. 3(c) and (d).

In order to obtain a quantitative characterization of the patterns we measure the correlations from a fixed site $i$ to any site $j$ as

$$
R(i, j)=\cos \left(\phi_{i}-\phi_{j}\right)
$$

and calculate the structure factor given by

$$
\begin{aligned}
& \mathbf{S}\left(k_{x}, k_{y}\right)=\sum_{i, j} R(i, j) \cos \left(k_{x} . i+k_{y} . j\right), \\
& \text { where } \frac{2 \pi}{N}<k_{x}, \quad k_{y}<2 \pi, \quad i, j:[1: N] .
\end{aligned}
$$

In this way we can define the structure factor as an order parameter, in a form that is similar to the tensor order parameter in nematic liquid crystals [31]. (a)
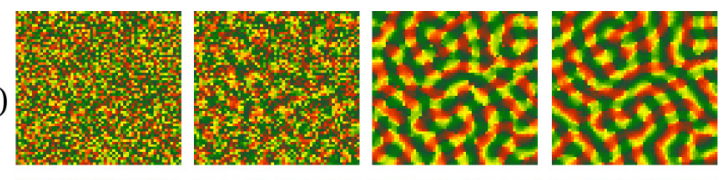

(b)
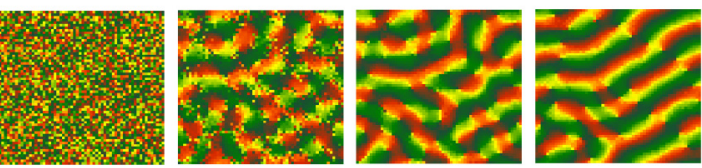

(c)
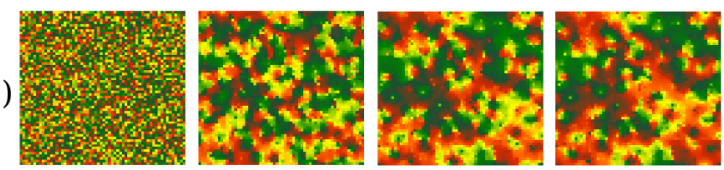

(d)
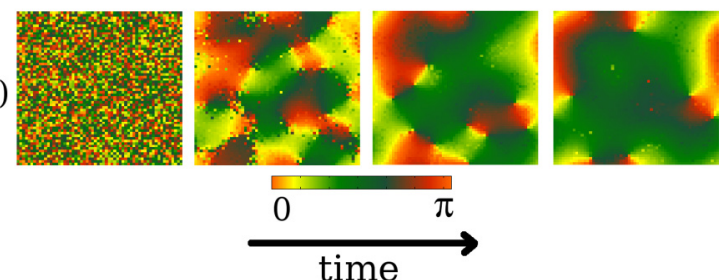

time

FIG. 3. (Color online) Temporal evolution of the phases for four different sets of parameters. The snapshots correspond to times $t=$ $0,25,50$, and $100 \mathrm{~s}$. The parameters for (a) and (b) are $A=10, \alpha=$ 2.1, $m=100, M=500, C=1$; additionally in (a) $\sigma^{2}=6$ and in (b) $\sigma^{2}=14$. For (c) $A=10, \alpha=2.5, m=4, M=400, C=1$, $\sigma^{2}=6$, and in (d) $A=10, \alpha=5, m=100, M=500, C=2$ and $\sigma^{2}$ is variable.

In Fig. 4 we show four stationary states of the system and their corresponding structure factors (the parameters are the same as in Fig. 3). For the striped phases in Figs. 4(a) and (b) the structure factor appears as a ring, whose radius and width is related to the width and spacing of the stripes. When there is no particular orientation preference of the stripes the ring is uniformly distributed. Later we will focus on the case when there is a clear orientation preference. For the clustered phases in Figs. 4(c) and (d) the structure factor presents a qualitatively different behavior, with a peak value at the origin surrounded by a fuzzy cloud. These different phases can be characterized quantitatively measuring and averaging the amplitudes of the wave vector in all directions. When a ring-like structure, that reveals the presence of stripes is present, a peak showing the width and radius of the ring can be clearly observed as in Fig. 5(a). On the other hand, for clustered phases, a single peak close to the origin followed by a fast decaying curve appears, as in Fig. 5(b).

This qualitative behavior strongly resembles the experimental results of Obermayer et al. [19]. They observed orientation preference in spatial patterns of infant macaques and made a Fourier analysis, which shows a clear ring link structure factor characterized by a single peak when plotted as a function of radial spatial frequency [19].

The presence of patterns with orientational preference can also be quantitatively characterized using the structure factor. In Fig. 6 we plot the values of the structure factor as a function of $k_{x}$ and $k_{y}$. Note the presence of two peaks, that reveal the orientational preference. 
(a)
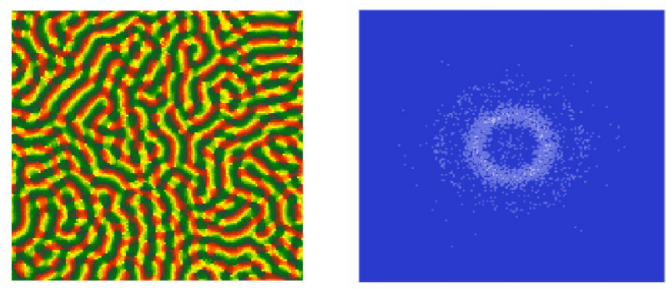

(b)

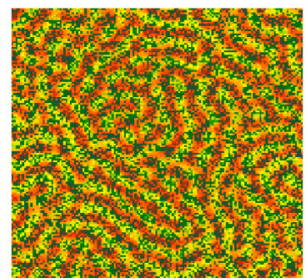

(c)

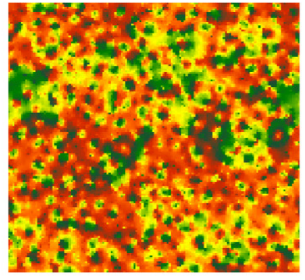

(d)
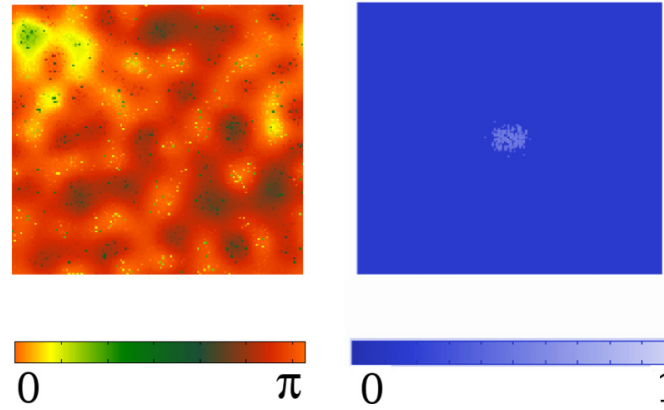

0
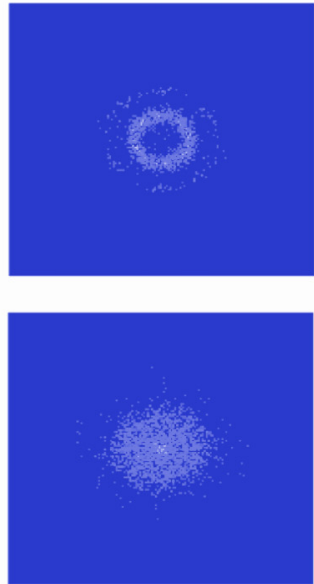

FIG. 4. (Color online) Snapshots of four stationary states of the system (left column) and their corresponding structure factors (right column). System size is $N=140 \times 140$ and parameters are the same as in Fig. 3.

In fact, if we measure the variation of the structure factor following $k_{x}$ the two peaks can be clearly observed, while an almost flat curve is present following $k_{y}$ Fig. 7 .

Using this quantitative information from the structure factor we are able to construct a phase diagram varying the Mexican hat parameters $C$ and $\sigma$. The line shows the position of the maximum of the mean value of the structure factor averaged over all directions as shown in Fig. 5. The phase diagram in Fig. 8 is divided then in two different phases, one with clustered and another with striped structures. For small values of $C$ and $\sigma$ clustered phases are present. As $\sigma$ is increased the system tends to a state with all phases synchronized, and the system seems to be dominated by the short range attractive interaction that tends to synchronize the phase oscillators. For a fixed value of $\sigma$, when $C$ is increased, a transition between clustered and striped phases takes place. In fact, increasing the value of $C$ allows for the competition between attractive and repulsive forces, a mechanism necessary for the formation of striped structures [32].
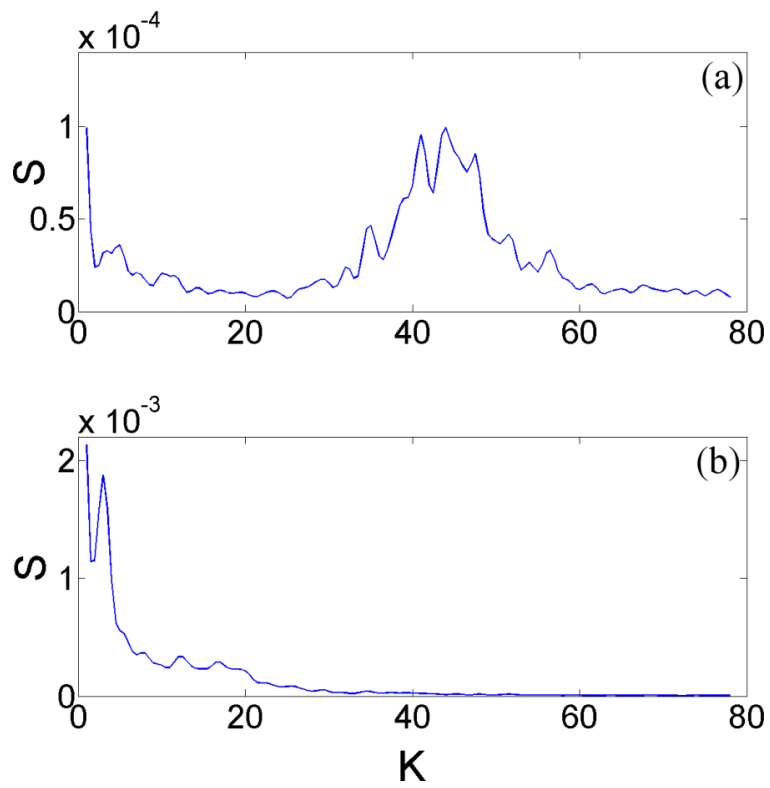

FIG. 5. (Color online) Mean value of the structure factor as a function of $k$ vector, averaged over all directions. Different qualitative behaviors corresponding to the striped phase (top curve) and clustered phase (bottom curve) can be clearly distinguished. System size is $N=140 \times 140$ and parameters are the same as in Fig. 3(a) and 3(c).

We have presented a model which has a strong biological inspiration, and in fact, as we will show, we are able to reproduce specific patterns that are observed in some species of mammals, referred as cardinal preference. In 1998, Coppola et al. [18] exposed a ferret to the usual moving bar stimuli and recorded the response of the cortex. Later, the image obtained was digitally processed in order to quantify the pixels corresponding to each preferred orientation and plotted in a histogram. The histogram obtained in this way revealed a major quantity of pixels in the horizontal and the vertical directions, reflecting a preference of the ferret cortex for these orientations.

We built histograms of the phases for parameters in the different regions of the phase diagram, and observed that it is possible to obtain patterns with cardinal preference. In Fig. 9 we present four different snapshots of the phases and their corresponding histograms. The figure clearly shows that increasing $C$ for a fixed value of $\sigma$ causes the systems to evolve from an almost uniform phase distribution to a bi-modal

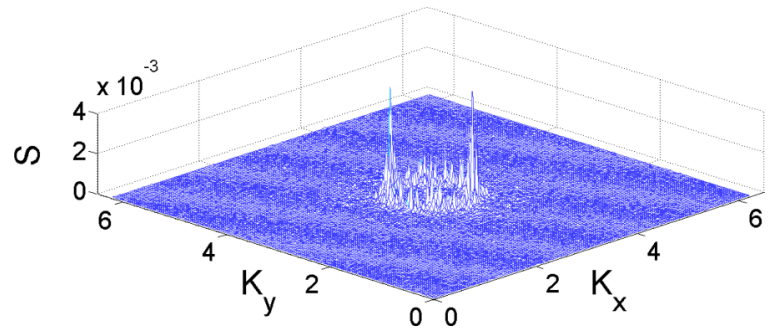

FIG. 6. (Color online) Structure factor as a function of $k_{x}$ and $k_{y}$ for parameters that presents a clear orientational preference characterized by the presence of two peaks that can be clearly distinguished. 

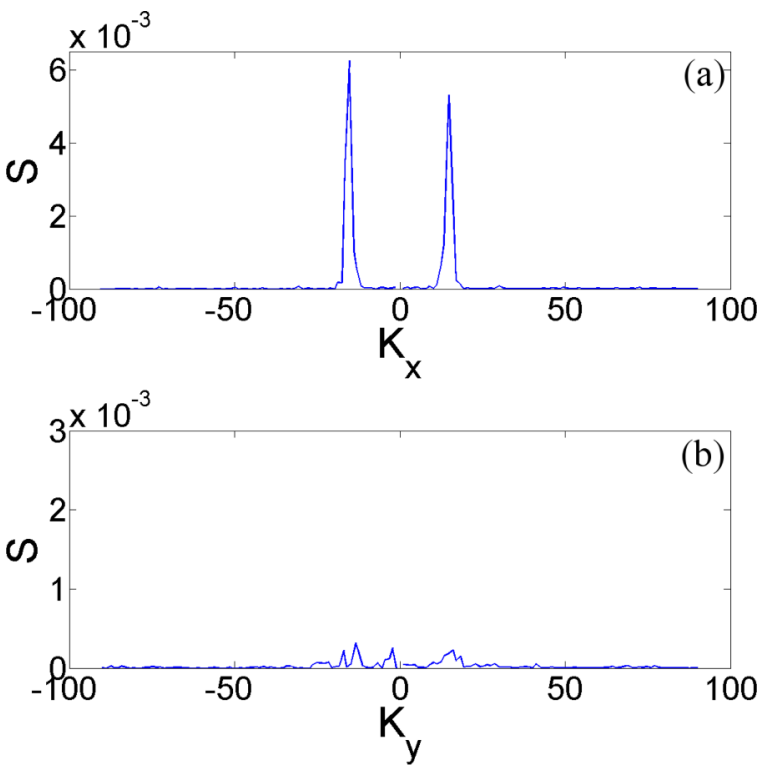

FIG. 7. (Color online) Mean value of the structure factor as a function of $k$ for two directions $\left(k_{x}, k_{y}\right)$. The curves reflect the difference between the strength of the structure factor in some directions for the case presented in Fig. 6.

distribution with a clear cardinal preference, that strongly resembles the experimental results of [18].

\section{DISCUSSION}

In this work we presented a model for pattern formation inspired in the behavior of the neurons in the visual cortex. It has been speculated that the functionality of these patterns is shaped by an economic trade-off between minimizing costs, such as connection lengths or processing time, while allowing for the emergence of topological patterns of anatomical or functional connectivity between multiple neuronal populations $[33,34]$. Along this line, in this work we presented a model which allows us to study the basic network and interaction mechanisms that allow for the emergence of patterns. In particular we considered phase oscillators coupled in an embedded complex network. Through the embedding we were able to take into account the geographical distance between the units in the interaction function. In contrast to other models of

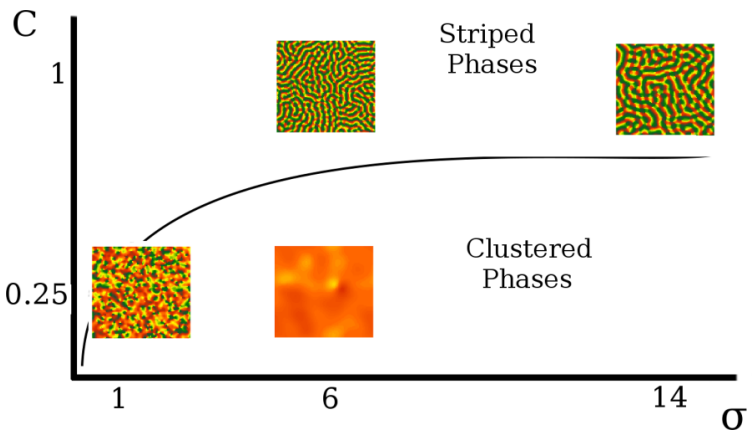

FIG. 8. (Color online) Phase diagram varying the Mexican hat parameters $C$ and $\sigma$. The transition between striped and clustered phases was determined quantitatively using the structure factor. (a)
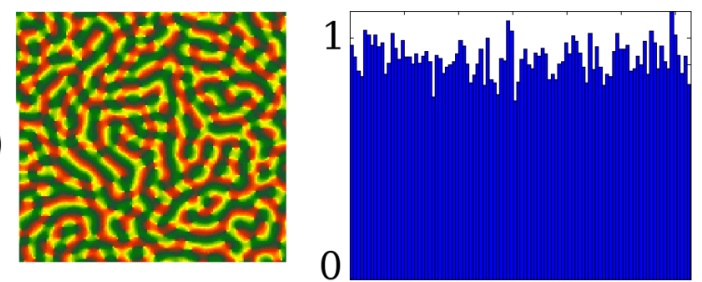

(b)
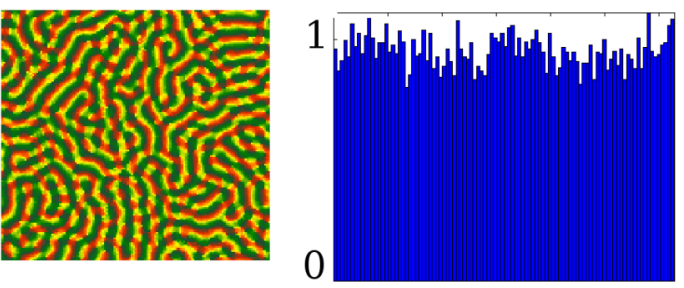

(C)
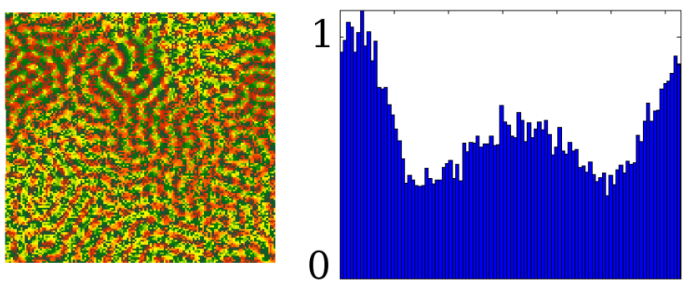

(d)
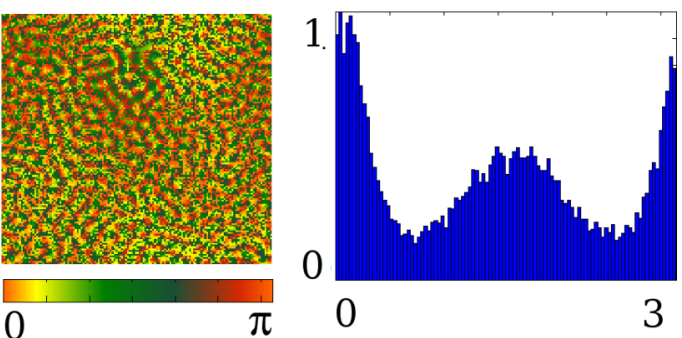

FIG. 9. (Color online) Histograms of the phases for $\sigma^{2}=6$ constant and varying $\mathrm{C}$ (from top to bottom $C=0.5,1.0,2.0$, and 3.0). The parameters used are $A=10, \alpha=2.1, m=100$, and $M=500$. System size is $N=140 \times 140$.

map formation where the connectivity between neurons and their synapses is distributed in layers [35,36], we considered a single layer, assuming that the response capacity varies laterally only and is approximately the same in all the layers [16]. Our results show that even with this approximation the model is still able to capture the main ingredients of the cortical organization. In fact, with only a few parameters, the model allows for the emergence of clustered or striped patterns.

We used the structure factor to quantitatively characterize the different patterns. This characterization allowed us to compare the numerical results with experimental data from infant macaques [19] and monkeys [20]. Also, using the structure factor we are able to construct a phase diagram in the variables that modulate the Mexican hat interaction function. We found two regions, one characterized by the presence of clustered phases, while the other presents striped phases. Finally, by tuning the parameter that modules the balance between inhibitory and excitatory interactions in the Mexican hat function, we were able to find striped structures with cardinal preference, resembling qualitatively experimental results observed by Coppola et al. [18] in ferrets. 
[1] G. Nicolis and C. Nicolis, Foundations of Complex Systems. Emergence, Information and Predicition (World Scientific, Singapore, 2012).

[2] S. Strogatz, The Emerging Science of Spontaneous Order (Hyperion, New York, 2003).

[3] S. Manrubia, A. Mikhailov, and D. Zannette, Emergence of Dynamical Order: Synchronization Phenomena in Complex Systems, Volume 2 of World Scientific Lecture notes in Complex Systems (World Scientific, Singapore, 2004).

[4] J. Murray, Mathematical Biology (Springer, Berlin, 1993).

[5] M. Newman, A. Barabási, and D. Watts, The Structure and Dynamics of Networks (Princeton University Press, Princeton, 2006).

[6] A.-L. Barabási and R. Albert, Science 286, 509 (1999).

[7] D. J. Watts and S. H. Strogatz, Nature 393, 440 (1998).

[8] S. Boccaletti, V. Latora, Y. Moreno, M. Chavez, and D.-U. Hwang, Phys. Rep. 424, 175 (2006).

[9] A. Arenas, A. D. Guilera, J. Kurths, Y. Moreno, and C. Zhou, Phys. Rep. 469, 93 (2008).

[10] P. M. Gleiser and D. H. Zanette, Eur. Phys. J. B 53, 233 (2006).

[11] I. J. G. Portillo and P. M. Gleiser, PLoS ONE 4, e6863 (2009).

[12] E. Bullmore and O. Sporns, Nature Reviews Neuroscience 10, 186 (2009).

[13] N. Swindale, Network: Comput. Neural Syst. 7, 161 (1996).

[14] K. Obermayer, G. G. Blasdel, and K. Schulten, Phys. Rev. A 45, 7568 (1992).

[15] K. Obermayer, K. Schulten, and G. G. Blasdel, Advances in Neural Information Processing Systems Vol. 4 (Morgan Kaufmann Publishers, San Francisco, CA, 1992), p. 83.

[16] E. Kandel, J. Schwartz, and T. Jensell, Principles of Neural Science (McGraw Hill Medical, New York, 2000).

[17] G. G. Blasdel and G. Salama, Nature 321, 579 (1986).
[18] D. M. Coppola, L. E. White, D. Fitzpatrick, and D. Purves, Proc. Natl. Acad. Sci. USA 95, 2621 (1998).

[19] K. Obermayer, L. Kiorpes, and G. G. Blasdel, Advances in Neural Information Processing Systems, Vol. 6 (Morgan Kaufmann Publishers, San Francisco, CA, 1994), p. 543.

[20] K. Obermayer and G. G. Blasdel, J. Neurosci. 13, 4114 (1993).

[21] Y. Kuramoto, Chemical Oscillations, Waves and Turbulence (Springer, Berlin, 1984).

[22] J. A. Acebrón, L. L. Bonilla, C. J. P. Vicente, F. Ritort, and R. Spigler, Rev. Mod. Phys. 77, 137 (2005).

[23] D. Hubel, "Eye, brain, and vision." http://hubel.med. harvard.edu/index.html.

[24] A. F. Rozenfeld, R. Cohen, D. ben-Avraham, and S. Havlin, Phys. Rev. Lett. 89, 218701 (2002).

[25] D. Ben-Abraham, A. Rozenfeld, R. Cohen, and S. Havlin, Physica A 330, 107 (2003).

[26] D. D. Stettler, A. Das, J. Bennett, and C. D. Gilbert, Neuron 36, 739 (2002).

[27] C. B. Tauro, F. A. Tamarit, and P. M. Gleiser, Physica A 391, 834 (2012).

[28] S. Kumar, Neural Networks: A Classroom Approach (McGrawHill Education, New York, 2004).

[29] T. Kohonen, Self-organization and Associative Memory, Springer Series in Information Sciences, 3rd ed. (Springer, Berlin, 1989).

[30] M. Cho and S. Kim, Phys. Rev. Lett. 92, 018101 (2004).

[31] D. A. Stariolo and D. G. Barci, J. Phys.: Conf. Ser. 246, 012021 (2010).

[32] M. Seul and D. Andelman, Science 267, 476 (1995).

[33] E. Bullmore and O. Sporns, Nature Reviews Neuroscience 13, 336 (2012).

[34] M. Kaschube, Current Opinion in Neurobiology 24, 95 (2014).

[35] S. Tanaka, Neural Networks 3, 625 (1990).

[36] S. Tanaka, Biol. Cybern. 64, 263 (1991). 